\title{
Models of approach to outpatient older persons care
}

\author{
Célia Pereira Caldas ${ }^{1, *}$, Renato Peixoto Veras ${ }^{2}$, Luciana Branco da Motta ${ }^{2}$, \\ Ricardo Carreño Siqueira ${ }^{2}$, Renata de Freitas Corrêa ${ }^{2}$, Marcelo de Jesus Carlos ${ }^{2}$, \\ Ana Carolina Lima Cavaletti Guerra ${ }^{2}$ \\ ${ }^{1}$ Third Age Open University / Rio de Janeiro State University, Rua São Francisco Xavier 524, Bloco F, Sala 10150, Maracanã - Rio de \\ Janeiro, RJ. Brazil. CEP 20559-900 \\ ${ }^{2}$ Third Age Open University/ Rio de Janeiro State University, Rio de Janeiro, RJ, Brazil
}

\section{Email address:}

celpcaldas@hotmail.com (C. P. Caldas), unativeras@gmail.com (R. P. Veras), lubmotta@gmail.com (L. B. Motta), rcs.carreno@gmail.com (R. C. Siqueira), renata.fcorrea@yahoo.com.br (R. d. F. Corrêa), marcelojcarlos@gmail.com (M.J. Carlos), carolinacavaletti@gmail.com (A. C. L. Cavaletti Guerra)

\section{To cite this article:}

Célia Pereira Caldas, Renato Peixoto Veras, Luciana Branco da Motta, Ricardo Carreño Siqueira, Renata de Freitas Corrêa, Marcelo de Jesus Carlos, Ana Carolina Lima Cavaletti Guerra. Models of Approach to Outpatient Older Persons Care. Science Journal of Public Health. Vol. 2, No. 5, 2014, pp. 447-453. doi: 10.11648/j.sjph.20140205.21

\begin{abstract}
Background: The aging of the population has generated discussions on the needs and unique characteristics of the users of health systems. In this context, the frailty has been used as a guide in managing health care for older adults and specific intervention has shown itself to be effective as much for the diagnosis of illnesses as the improved functioning and satisfaction of the patient and the reduction of mortality. Aims and objetive: The aim of this study was to become familiar with approaches to outpatient older people care for frail older adults. A critical review was conducted evaluating the effectiveness of these models and researchers looked for methods developed in outpatient facilities by interprofessional teams. Conclusions: The models that met our criteria for eligibility presented interprofessional teams composed of geriatricians, nurses, social workers, physiotherapists, occupational therapists, speech therapists, nutritionists and pharmacists. All of the models offered managed care of their patients and the professional who carry out these tasks are mainly nurses, but also social workers, or a primary care physician. Relevance to Clinical Practice: Our results showed that the configuration of a specialized outpatient model in the care of the frail older person is a recent phenomenon, with benefits such as reduced polypharmacy and decreases in functional loss, resulting in a greater quality of life for the users.
\end{abstract}

Keywords: Outpatient Care, Frailty, Interdisciplinary Health Teams, Ambulatory Health Center

\section{Introduction}

The aging of the population has generated discussions on the needs and unique characteristics of the older adults as users of health systems. Concern with the quality of health care offered to the older persons using the resources available within the health systems in a sustainable manner is indeed intense [1, 2, 3, 4].

It is common to encounter among older patients reports of frailty, functional and cognitive decline, loss of autonomy, polypharmacy, and coexistence of morbidities [5]. Concomitant the incidence of admissions is high in emergency in many countries, needing appropriateness of admissions and practices preventive and efficient in community [6].
Frailty in the older people has been identified as risk indicator of death or events that may alter their autonomy. Consequently it is used as a guide in managing health care $[7,8,9]$.

In this context, specialized intervention has shown itself to be effective as much for the diagnosis of illnesses as the improved functioning and satisfaction of the patient and the reduction of mortality [10].

Although smaller in magnitude when compared to hospitalized patients, there is evidence for the effectiveness of specialized assessment interventions and care management (GEM - Geriatric Assessment and Management) on an outpatient basis for the functional preservation and amelioration of frail adults residing in the community $[11,12]$.

To preserve independence and prevent functional disabilities, proactive health programs are recommended. 
These programs should be integrated and structured in a multidisciplinary approach and accompanied by care management. They should also encourage physical activity for individuals with moderate physical frailty $[13,14,15]$.

The guiding question for the present study is which approaches to older people outpatient care for the frail adults are effective. In this review of the literature we are looking for information on structure, dynamics, work processes, and evaluation of the effectiveness of the interprofessional approach in outpatient units.

\section{Objectives and Methods}

\subsection{Identification of Studies}

The electronic bibliographic databases searched were: PubMed via the National Center for Biotechnology Information (NCBI); SCOPUS and Web of Science via the Brazilian Research Agency Journals Website (CAPES); EMBASE; The Cochrane Library, LILACS, PAHO, WOLIS MEDCARIBE E IBECS via Health Virtual Library (BVS); and Scientific Electronic Library Online (Scielo).

The descriptors used for all the databases were: Ambulatory Care, Health Services for the Aged, geriatric assessment, Structure of services, Delivery of health care, Integrated care, frail older persons, program evaluation, effectiveness, health evaluation.

We did not limit our search by date of publication but included publications until July 2013, and the text of the articles had to be available in English, Spanish or Portuguese. At this stage we also did not limit our search by type of study.

\subsection{Criteria for the Selection of Studies}

Five researchers conducted identification of the studies independently, in such a manner that two researchers searched each database. The studies had to meet some preestablished criteria for inclusion in the review. Regarding the population, the individuals should be equal to or greater than sixty years old, previously considered frail, recognized as having multiple pathologies requiring monitoring for chronic illness or in a period immediately following hospital discharge. The researchers looked for methods developed in outpatient facilities by interprofessional teams. Methods in emergency units, homes, and among hospitalized or institutionalized patients were excluded from the review.

After being identified, the summaries of the pre-selected articles were reviewed by three researchers independently. Each researcher re-applied the selection criteria and verified the presence of an analysis of effectiveness.

Selected for full reading were studies that were unclear as to the interprofessional approach in an outpatient facility or the presence of an evaluation of effectiveness.

\subsection{Summary of the Information}

Two researchers conducted a reading of the full text of the articles individually, making use of a third reviewer in cases of a discrepancy in the eligibility criteria. Using a standardized and prepared form, each investigator extracted the following data from each eligible study: type of study, population, methodological description, period and location of the study, study aims, subjective quality of the study, professionals involved in the approach, description of the approach, results and a space for individual comments from the investigator, for instance regarding study biases.

\section{Results}

\subsection{Included Studies}

Search five retrieved a total of 18,388 citations, of which 282 studies were pre-selected after a reading of the title and summary. After removing duplicates $(n=120)$, the abstract of potentially relevant papers were reviewed. After a second selection process remaining 33 articles for detailed reading. Figure 1 illustrates the process of selection and eligibility of the articles.

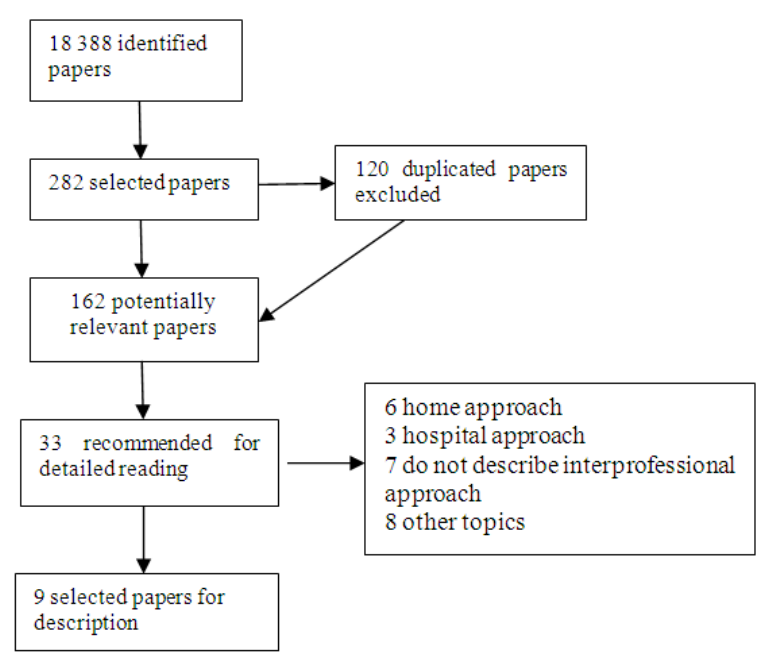

Fig. 1. flowchart of the selection of studies

The research methodology utilized was intended to produce a systematic revision of the literature on ambulatory practices to serve the frail older persons. However, most clinical trials that meet our eligibility criteria are still in progress. Thus, we present the description of the chosen model practices, considering other forms of study together with results of the analysis of effectiveness, when available.

The study that demonstrated the best relation with our objectives was conducted in the city of Ramsay, in Minnesota (USA). In a randomized clinical trial conducted by Boult [16] an attempt was made to transfer the use of the CGA (Comprehensive Geriatric Assessment) with interprofissional monitoring at hospital levels to the outpatient clinic. The study evaluated 586 persons older than 70 and with risk of repeat internment according to the PRA screening instrument, a method developed by the same author. After randomization the primary care 
physicians were notified of the frailty of the persons in the control group and the intervention group received integrated support from the social workers, nurse and physician.

The intervention group passed through four stages. The first, obtain permission from the medical assistant. Later, they received a home visit from a social worker and went twice to a GEM (Geriatric Evaluation and Management) clinic. The first was for an evaluation by a nurse from the group (for a physical exam) and the second for attendance from the geriatrician together with the nurse to assess the medical and psychosocial conditions, functional capacity, cognitive state, social network, gait and balance, and the safety of the immediate environment [16].

Based on this plan the intervention group received care from GEM professionals during 6 months. Afterwards, a brief and incomplete evaluation regarding the costs was undertaken.

Evaluation of the results was conducted by functional tests and symptoms of depression. Patients were compared after six, twelve and eighteen months. As expected by the author, initial Medicare expenditures by the intervention group were higher in the first six months, however after eighteen months Medicare costs were 3.8\% higher for the control group. The costs analysis was restricted to Medicare and is therefore not statistically significant.

In the analysis of functionality, the intervention group showed no progression of functional loss in comparison with the control group in the three instances of measurement. Screening for depressive symptoms accompanied these positive results only after 12 months, with maintenance after 18 months. Higher satisfaction rates were also perceived among the users in the intervention group [16].

Although this study shows no progression in functional loss, the study fails to demonstrate a decrease in costs, by its measurements, and likewise of mortality. However the positive results may be underestimated due to the individual efforts of the control group's outpatient physician to improve the situation of his patient when notified of the patient's level of risk.

Another study [17] proposes a model whose aim is to improve the management of the following geriatric syndromes: urinary incontinence, depressive symptoms, falls, use of high-risk medications and functional decline. The "Chronic Disease Score", a computerized predictive index, was used with 50 thousand patients 65 years or older in Washington DC, from which 324 were selected and only 173 were used in the study.

After randomization, the intervention group was assessed every 3-4 months by an interprofessional team consisting of pharmacists, physicians, nurses, and social workers. The intervention consisted of a 30 minute consultation with a physician or nurse to develop a treatment plan with an emphasis on reducing disabilities; a 15 minute consultation with a pharmacist to check for medications associated with functional decline and polypharmacy; a 45 minute session on self-management lead by a nurse or social worker, involving physical exercise, nutrition and advanced care; and finally information about the health assessment offered by the professional team during the visit. The control group was distributed into groups monitored by 4 physicians. The groups were compared at the beginning, and again after 12 and 24 months [17].

At 12 months the control group had more symptoms of urinary incontinence, but not at 24 months. This was the only difference in the analysis of geriatric syndromes [17]. This study had several limitations and failed to show any benefits in the control of geriatric syndromes or costs. However, the intervention group demonstrated a higher level of user satisfaction.

Lois K. Evans and collaborators [18] describe a model of care management by nursing professionals. It consists of collaboration between these nurse managers, physicians, physiotherapists and occupational therapists. This model describes 2 or 3 consultations per week during a 2 to 9 week period, at the discretion of the team. With the aim of greater efficiency in providing services, the care manager integrates and coordinates care among the health professionals and support network [18]. This study also has a descriptive character and repeats a trend in the literature that studies health models for the older persons in which a health professional is introduced to assume the management of care. Nonetheless there is not yet a consensus on what should be the professional attributions, level of intervention and qualifications of this manager.

A model of outpatient attendance for frail seniors was developed at a tertiary medical center in Taipei, Taiwan. The model uses Comprehensive Geriatric Assessment interventions and Geriatric Evaluation and Management in a multidisciplinary service with health care assistance carried out by various physicians (general practitioner or family doctor, geriatric psychiatrist, physiatrist and geriatrician). They offer physiotherapy and occupational therapy interventions in addition to pharmacological treatment. First the patient's general practitioner or family doctor conducts a complete medical assessment and determines if an CGA is required [19].

Next, a trained nurse conducts the CGA in the selected patients. After obtaining all the information, the geriatrician consults with the elder patient, outlining a care plan and determines treatment goals. According to the case, the geriatrician may discuss the care plan with the physiatrist and the psychiatrist. The monitoring service is offered twice per week, according to the consultations limits imposed by the reimbursement regulations of the national health insurance (National Health Insurance) [19].

The effectiveness of this service model was evaluated by indicators of medication use and by quality of life measures. The study was prospective with before and after analysis conducted in 2007 and 2008. The study inclusion criteria were: (1) greater than 80 years old and in any health condition; (2) 65 or older with more than three comorbidities; (3) 65 or older with established geriatric 
syndrome.

The participants were monitored by telephone by a nurse every three months during the 12 months duration of the study. At the end of the follow-up a brief CGA was conducted by the same professional who performed the quarterly monitoring of the patient [19].

One hundred and thirty-five patients completed the study. They registered a small but significant reduction in the use of oral medication $(3.8 \pm 2.8$ items vs. $3.2 \pm 2.7$ items, $\mathrm{p}<0.001$ ) during the period of the study, in addition to a significant improvement in the quality of life $(0.65 \pm 0.08$ vs. $0.62 \pm 0.04, \mathrm{p}<0.001)$. The quality of life acquired with this model was estimated to be 4.1 years [19]. The study presents important limitations, among them the fact that it is not a randomized clinical trial and they did not conduct a cost-effectiveness analysis [19].

In what follows we cite texts that, despite not meeting our eligibility criteria, offer processes or concepts that can assist in the development of an interprofessional older people care model.

A study conducted at the University of North Carolina in the United States [20] endeavors to contribute to the formulation and expansion of models of assessment in an attempt to improve the exploration of results in the older population. Employing a "holistic" vision of the patient, an interprofessional team uses a working method described as "trans disciplinary"- despite the fact that the team used in the study was described as "interdisciplinary".

The existing model in pediatrics was adapted for geriatrics, in which the entire team meets in a room where the assessment takes place, with the possibility of exchanging or reducing professionals to tackle specific issues. In this assessment preparation and explanation is conducted together with the patient and family input in order to gather together and acknowledge their demands and finally to elaborate a treatment plan [20]. This is a descriptive study and does not offer any kind of evaluation of effectiveness. It does however show an aspect of a model in which an interprofessional team is integrated in a unique manner in an attempt to better assess the health status of the patient.

The Walcheren Integrated Care Model (WICM) [21] is a Dutch model for caring for the health of frail seniors. This model is part of a national program for older persons care, the National Care for the Elderly Program. It was developed to offer complete care to frail older adults using primary care outpatient structure, the General Practitioner practice. The aim is to improve the quality and efficiency of care offered by caregivers and health professionals to frail older persons living independently [21].

Among the key elements of the WICM are: the General Practitioner practice as the single point of entry; stratification/risk screening (detection of frailty); multidimensional instrument of assessment based on evidence; individualized multidisciplinary plan; case management; meeting and consultations with a multidisciplinary staff; care protocols; orientation group; specialization and delegation of tasks; and a computerized information system [21].

According to the authors, the selection of eligible patients will be performed by screening the frailty of seniors 75 or older, cared for by primary care physicians (General Practitioners), using the Groningen Frailty Indicator screening instrument, a questionnaire composed of 15 items that measures functional decline in 4 domains: physical, cognitive, social and psychological. A nurse with experience together with a primary care physician calculates the Groningen Frailty Indicator score. Once identified, the frail persons are assigned to a case manager. This care management is carried out by a specialized or trained nurse, in the case of persons with more complex needs [21].

The case manager then performs the multidimensional EASY Care assessment and prepares and individual care plan that is subsequently discussed in a meeting with the GP (General Practitioners) and a multidisciplinary team. This team is composed of geriatricians, in-home care professionals, paramedics, social workers, pharmacists and mental health professionals. During this meeting a multidisciplinary care plan is developed based on the treatment plan of each professional on the team [21].

After the individualized multidisciplinary care plan is developed the GP shares it with the patient and his or her caregivers in order to obtain permission for its implementation. The case manager is responsible for admitting the patient into the necessary services, planning and coordinating the care offered, and should also monitor the patient every six months, in addition to periodically reevaluating the care plan. This nurse is also the one who supports the multidisciplinary team by organizing meetings and facilitating the exchange of information [21].

Regarding the professional skills requirements for this model, the GP should have training in geriatrics; the case manager should have a degree in case management, and both should have completed training in EASY Care [21]. The WICM has yet to be implemented or evaluated; the article is a study protocol and does not present results.

Another study protocol describes an interprofessional care model at an Ambulatory Geriatric Unit in Norrköping, Sweden, composed of physicians, nurses, physiotherapists, occupational therapists, nutritionists, social workers, and pharmacists [22].

The randomized and controlled clinical trial will have 198 participants in each group (control and intervention groups), aged 75 or older who have been hospitalized at least three times in the 12 months preceding the study, or who have three or more diagnoses according to the CID 10 [22].

After selection a nurse performs an initial assessment (CGA). Twice a week a team meets for 30-60 minutes to discuss the patient's situation. The physical, psychological, social and functional conditions are monitored to and the physician determines actions such as adjustments in the medication and the pharmaceutical approach, the need for 
domestic visits, and physic- or occupational therapy in an endeavor to increase the patient's quality of life. The intention is to analyze the effectiveness of the model through the number of hospitalizations and changes in the stages of frailty [22].

In Toulouse, France, a platform was developed for the assessment of frailty and disability prevention among the frail older persons receiving primary care [23]. This health care model is anchored in a multidisciplinary team and primary care that is the single point of entry to the health care system. The model proposes preventive and therapeutic interventions, family and caregiver support, and interaction with the primary care physician with the goal of optimizing the frail patient's care management.

The service, initiated in October 2011, is covered by the national health insurance system and operates at the Geriatric Day Hospital of the geriatric unit of the Hospital Garonne, called the Gérontopôle de Toulouse. The platform initially accommodated up to four patients per day, five days per week, and there was an expected increase in capacity up to eight patients per day starting in January 2013 [23].

Screening of the frail older persons is performed by a primary care physician using a frailty screening instrument - devised by them based on Fried's criteria with the additional perception of the GP - which is applied with patients 65 or older [23].

The first approach is a multidimensional assessment conducted by a geriatric physician (or GP with a background in geriatrics) and a nurse. Complementary exams are also performed. According to the needs, other professionals may evaluate the patient: neuropsychiatries, ophthalmologist, nutritionist, physiotherapist, dentist and social worker [23].

Based on this multidisciplinary assessment, the geriatrician elaborates a personalized care plan that is proposed and discussed by telephone with the patient's primary care physician. The patient continues to be seen by his or her primary care physician and, in order to increase effectiveness and adherence to the care plan, the patient is also monitored by a platform nurse by telephone regularly. In case of functional decline, new actions are discussed after contacting the patient's physician and a reevaluation by the physician [23].

There is no study yet of effectiveness. According to the authors the next objective is to conduct an evaluation of cost-effectiveness and clinical effectiveness, in particular for the prevention of disabilities.

In Israel a model of case management was tested among participants in the Israel Long-Term Care Insurance program (LTC Law). The Social Services office of the Department of Well-Being for the city of Haifa was chosen to try out the project, which began at the end of March 1999. The case managers were two social workers from the Social Services office dedicating 10 hours per week [24].

The proposed intervention included referral, admission and assessment of needs; activation of a care plan with the services of the community; link services such as hospital and volunteers; monitoring of the quality of services offered; reassessment of needs and evaluation of outcomes [24].

Through home visits to patients once a week, the case managers discuss the care plan with the patient and family in order to adapt to values and preferences. They attended meetings with the interdisciplinary team ever two weeks and reviewed the patient's assessment and care plan every two months [24].

Other activities for the case managers in this model were to meet with staff and management of the Day Center where the participant was attended and trained by volunteers from the Volunteer Assistance Center in activities such as how to cook, administer medications, clean clothes, iron, clean the house, take walking and shopping [24].

\section{Discussion and Conclusion}

In the development of our work we had difficulty finding studies on outpatient models specializing in geriatrics. We encountered mostly hospital and in-home geriatric approaches. One hypothesis is that the centers specializing in geriatrics have not yet published on their work process or the cost-effectiveness of their services. Due to this difficulty, articles were selected that describe interprofessional approaches to the health care of frail older people in an outpatient setting.

An important finding is that the services of a multidisciplinary geriatric assessment are usually performed at home or in the hospital [25, 26, 27], not necessarily by a team, but individually by professionals from diverse specializations, or even just a nurse or doctor.

Case management is generally performed in-home by a nurse, social worker or physician [15, 28, 29, 30, 31]. Here we highlight the lack of consensus on the appropriate professional to exercise this function, what tasks he or she should assume, and the cost effectiveness of including this manager in the frail older people care model.

Regarding the medical professional member of the team, for the in-home model generally in doctor is a primary care physician $[15,30,31]$ and for the hospital model usually a geriatrician [25]. For the outpatient facilities model we found doctors working in close collaboration with nurse gerontologists [18] or a geriatrician as a member of the team $[16,19]$.

The multidisciplinary team in the outpatient model mainly consists of a physician or geriatrician, nurse and social worker $[16,17,18]$ together with physiotherapists, occupational therapists, speech therapists, nutritionists and pharmacists $[18,19]$.

We have observed that in health systems based on primary care, the frail person receive in-home care when they have adequate social support or in long-term institutions. In other systems they receive emergency home visits or are taken to emergency hospitals when necessary.

Our conclusion is that structuring an outpatient model 
specializing in care for the frail older person is a recent phenomenon, offering benefits such as a reduction in polypharmacy and functional loss and resulting in a better quality of life for the users. More studies are necessary to verify the effectiveness in relation to costs in the implementation of these models.

\section{References}

[1] C. Bielaszka-DuVernay, "The 'GRACE' model: in-home assessments lead to better care for dual eligible," Health Affairs (Project Hope), vol. 30, pp. 431-434, 2011.

[2] S. R. Counsell, C. M. Callahan, D. O. Clark, W. Tu, A. B. Buttar, T. E. Stump, G. D. Ricketts, "Geriatric care management for low-income seniors: a randomized controlled trial," JAMA: The Journal of the American Medical Association, vol. 298, pp. 2623-33, 2007.

[3] R. A. Lourenço, C. S. F. Martins, M. A. S. Sanchez, R. P. Veras, "Assistência ambulatorial geriátrica: hierarquização da demanda," Revista de Saúde Pública, vol. 39, pp. 311$318,2005$.

[4] R. P. Veras, "Gerenciamento de doença crônica: equívoco para o grupo etário dos idosos," Revista de Saúde Pública, vol. 46, pp. 929-934, 2012.

[5] G. Abellan Van Kan, A. Sinclair, S. Andrieu, M. Olde Rikkert, G. Gambassi, and B. Vellas, "The geriatric minimum data set for clinical trials (GMDS)," The Journal of Nutrition, Health \& Aging, vol. 12, pp. 197-200, 2008.

[6] B. Walsh, H. Roberts, J. Hopkinson, "Emergency hospital admissions for ill-defined conditions amongst older people: a review of the literature," International journal of older people nursing, vol. 2, pp. 270-7, 2007.

[7] J. McCusker, J. Verdon, P. Tousignant, L. P. de Courval, N Dendukuri, E. Belzile, "Rapid emergency department intervention for older people reduces risk of functional decline: results of a multicenter randomized trial," Journal of the American Geriatrics Society, vol. 49, pp. 1272-81, 2001 .

[8] R. E. Pel-Littel, M. J. Schuurmans, M. H. Emmelot-Vonk, H. J. J. Verhaar, "Frailty: defining and measuring of a concept," The Journal of Nutrition, Health \& Aging, vol. 13, pp. 3904, 2009.

[9] J. Walston, E. C. Hadley, L. Ferrucci, J. M. Guralnik, A. B. Newman, S. A. Studenski, W. B. Ershler, T. Harris, L. P. Fried, "Research agenda for frailty in older adults: toward a better understanding of physiology and etiology: summary from the American Geriatrics Society/National Institute on Aging Research Conference on Frailty in Older Adults," Journal of the American Geriatrics Society, vol. 54, pp. 991$1001,2006$.

[10] J. Ploeg, J. Feightner, B. Hutchison, C. Patterson, C. Sigouin, M. Gauld, "Effectiveness of preventive primary care outreach interventions aimed at older people: meta-analysis of randomized controlled trials," Canadian Family Physician Médecin de Famille Canadien, vol. 51, pp. 1244-5, 2005.

[11] N. J. Cordato, S. Saha, M. A. Price, "Geriatric interventions: the evidence base for comprehensive health care services for older people," Australian Health Review: A Publication of the Australian Hospital Association, vol. 29, pp. 151-5, 2005.

[12] E. Rosted, L. Wagner, C. Hendriksen, I. Poulsen, "Geriatric nursing assessment and intervention in an emergency department: a pilot study," International Journal of Older People Nursing, vol. 7, pp. 141-151, 2012.

[13] S. Eloranta, S. Arve, P. Routasalo, "Multiprofessional collaboration promoting home care clients' personal resources: perspectives of older clients," International journal of older people nursing, vol. 3, pp. 88-95, 2008.

[14] R. Daniels, S. Metzelthin, E. Rossum, L. Witte, W. Heuvel, "Interventions to prevent disability in frail communitydwelling older persons: an overview," European Journal of Ageing, vol. 7, pp. 37-55, 2010.

[15] N. Bleijenberg, I. Drubbel, V. H. Ten Dam, M. E. Numans, M. J. Schuurmans, N. J. de Wit, "Proactive and integrated primary care for frail older people: design and methodological challenges of the Utrecht primary care PROactive frailty intervention trial (U-PROFIT)," BMC Geriatrics, vol. 12, pp. 16, 2012.

[16] C. Boult, L. B. Boult, L. Morishita, B. Dowd, R. L. Kane, C. F. Urdangarin, "A randomized clinical trial of outpatient geriatric evaluation and management," Journal of the American Geriatrics Society, vol. 49, pp. 351-359, 2001.

[17] E. A. Coleman, L. C. Grothaus, N. Sandhu, E. H. Wagner, "Chronic care clinics: a randomized controlled trial of a new model of primary care for frail older adults," Journal of the American Geriatrics Society, vol. 47, pp. 775-83, 1999.

[18] L. K. Evans, J. Yurkow, E. L. Siegler, "The CARE Program: a nurse-managed collaborative outpatient program to improve function of frail older people. Collaborative Assessment and Rehabilitation for Elders," Journal of the American Geriatrics Society, vol. 43, pp. 1155-60, 1995.

[19] M.-H. Lin, C.-L. Liu, L.-N. Peng, Y.-T. Chen, L.-K. Chen, "Demographic characteristics and clinical benefits of outpatient geriatric evaluation and management service in Taiwan," Archives of Gerontology and Geriatrics, vol. 55, pp. 42-4, 2012.

[20] S. Coppola, C. A. Rosemond, N. Greger-Holt, F. G. Soltys, L. C. Hanson, M. A. Snider, J. Busby-Whitehead, "Arena assessment: evolution of teamwork for frail older adults," Top Geriatr Rehabil, vol. 17, pp. 13-28, 2002.

[21] I. N. Fabbricotti, B. Janse, W. M. Looman, R. de Kuijper, J. D. H. van Wijngaarden, A. Reiffers, "Integrated care for frail elderly compared to usual care: a study protocol of a quasi-experiment on the effects on the frail elderly, their caregivers, health professionals and health care costs," BMC Geriatrics, vol. 13, pp. 31, 2013.

[22] A. L. Mazya, J. Eckerblad, T. Jaarsma, I. Hellström, B. Krevers, A. Milberg, M. Unosson, A. Westöö, A. Ekdahl, "The Ambulatory Geriatric Assessment - a Frailty Intervention Trial (AGe-FIT) - a randomised controlled trial aimed to prevent hospital readmissions and functional deterioration in high risk older adults: a study protocol," European Geriatric Medicine, pp. 242-247, 2013.

[23] J. Subra, S. Gillette-Guyonnet, M. Cesari, S. Oustric, B. Vellas, "The integration of frailty into clinical practice: preliminary results from the Gérontopôle," The Journal of Nutrition, Health \& Aging, vol. 16, pp. 714-20, 2012. 
[24] A. Lowenstein, "A case management demonstration project for the frail elderly in Israel," Care Management Journals : Journal of Case Management; The Journal of Long Term Home Health Care, vol. 2, pp. 5-14, 2000.

[25] H. H. Handoll, I. D. Cameron, J. C. Mak, T. P. Finnegan, "Multidisciplinary rehabilitation for older people with hip fractures," The Cochrane Database of Systematic Reviews, vol. 7, pp.CD007125, 2009.

[26] H. Hasson, S. Blomberg, A. Dunér, "Fidelity and moderating factors in complex interventions: a case study of a continuum of care program for frail elderly people in health and social care," Implementation Science: IS, vol. 7, pp. 23-34, 2012.

[27] M. E. Keough, T. S. Field, J. H. Gurwitz, "A model of community-based interdisciplinary team training in the care of the frail elderly," Academic Medicine: Journal of the Association of American Medical Colleges, vol. 77, pp. 936, 2002.

[28] N. Fairhall, C. Aggar, S. E. Kurrle, C. Sherrington, S. Lord,
K. Lockwood, N. Monaghan, I. D. Cameron, "Frailty Intervention Trial (FIT)," BMC Geriatrics, vol. 8, pp. 27, 2008.

[29] R. J. F. Melis, E. Adang, S. Teerenstra, M. I. van Eijken, A. Wilmo, T. van Achterberg, E. H. van de Lisdonk, M. G. Rikkert, "Cost-effectiveness of a multidisciplinary intervention model for community-dwelling frail older people," The Journals of Gerontology. Series A, Biological Sciences and Medical Sciences, vol. 63, pp. 275-282, 2008.

[30] M. E. Muntinga, E. O. Hoogendijk, K. M. van Leeuwen, H. P. J. van Hout, J. W. R. Twisk, H. E. van der Horst, G. Nijpels, A. P. D. Jansen, "Implementing the chronic care model for frail older adults in the Netherlands: study protocol of ACT (frail older adults: care in transition)," BMC Geriatrics, vol. 12, pp. 19-29, 2012.

[31] N. Van den Berg, C. Meinke, M. Matzke, R. Heymann, S. Flessa, W. Hoffmann, "Delegation of GP-home visits to qualified practice assistants: assessment of economic effects in an ambulatory healthcare centre," BMC Health Services Research, vol. 10, pp. 155-163, 2010. 